\title{
A!
}

This is an electronic reprint of the original article.

This reprint may differ from the original in pagination and typographic detail.

Tammelin, T.; Abburi, R.; Gestranius, M.; Laine, C.; Setälä, H.; Österberg, Monika

Correlation between cellulose thin film supramolecular structures and interactions with water

Published in:

Soft Matter

DOI:

$10.1039 / \mathrm{c} 5 \mathrm{sm} 00374 \mathrm{a}$

Published: 01/01/2015

Document Version

Peer reviewed version

Please cite the original version:

Tammelin, T., Abburi, R., Gestranius, M., Laine, C., Setälä, H., \& Österberg, M. (2015). Correlation between cellulose thin film supramolecular structures and interactions with water. Soft Matter, 11(21), 4273-4282.

https://doi.org/10.1039/c5sm00374a

This material is protected by copyright and other intellectual property rights, and duplication or sale of all or part of any of the repository collections is not permitted, except that material may be duplicated by you for your research use or educational purposes in electronic or print form. You must obtain permission for any other use. Electronic or print copies may not be offered, whether for sale or otherwise to anyone who is not an authorised user. 


\title{
ARTICLE
}

Cite this: DOI: 10.1039/x0xx00000x

\section{Correlation between cellulose thin film supramolecular structure and interactions with water}

\author{
Tekla Tammelin ${ }^{a *}$, Ramarao Abburib ${ }^{\mathrm{b}}$, Marie Gestranius ${ }^{\mathrm{a}}$, Christiane Laine ${ }^{\mathrm{a}}$, Harri \\ Setäläa , Monika Österberg ${ }^{b *}$
}

Received 00th January 2012,

Accepted 00th January 2012

DOI: $10.1039 / \times 0 \times x 00000 x$

www.rsc.org/

\begin{abstract}
Water interactions of ultra-thin films of wood-derived polysaccharides were investigated by using surface sensitive methods, Quartz Crystal Microbalance with Dissipation (QCM-D) and Atomic Force Microscopy (AFM). This approach allows systematic molecular level detection and reveals the information of biobased materials' inherent behaviour with nanosensitivity. The influence of structural features of cellulose films i.e. crystallinity, surface roughness and porosity on water interactions were clarified. Cellulose films were prepared using spin-coating and Langmuir-Schaefer deposition to obtain thin films of equal thickness, identical cellulose origin, simultaneously with different supramolecular structure. The uptake/release of water molecules and swelling were followed using QCM-D, and the structural features of the films were evaluated by AFM. More crystalline cellulose film possessed nanoporosity and as a consequence higher accessible surface area (more binding sites for water) and thus, it was capable to bind more water molecules in humid air and when immersed in water when compared to amorphous cellulose film. Due to the ordered structure, more crystalline cellulose film remained rigid and elastic although the water binding ability was more pronounced compared to amorphous film. Lower amount of bound water induced softening of the amorphous cellulose film and elastic layer became viscoelastic at high humidity. Finally, cellulose thin films were modified by adsorbing a layer of 1-butyloxy-2-hydroxypropyl xylan, and the effect on moisture uptake was investigated. It was found that the supramolecular structure of the cellulose substrate has effect not only on the adsorbed amount of xylan derivative but also on the water interactions of the material.
\end{abstract}

\section{Introduction}

Cellulose and hemicelluloses can be regarded as the most abundant plant based resource since they are the main structural cell wall components comprising up to $75 \mathrm{w}-\%$ of cell wall dry solids content. The main drivers for utilization of plant based resources for novel application areas such as packaging barrier materials, (bio)sensors, OLED displays and selective membranes have been the sustainability trend, fluctuating price of petroleum as well as unique features especially related to permeation and diffusion characteristics of oxygen and water molecules through these materials. ${ }^{1-10}$

The gas barrier performance of the material is defined in terms of diffusivity and solubility of permeant gas by mechanism which applies to gas transport through materials. ${ }^{11}$ Hydrophilic polysaccharide based polymer films which contain large amount of highly polar hydroxyl groups have an ability to efficiently limit the diffusion and permeability of oxygen molecules especially in dry conditions due to the low polarity of oxygen. At high humidity highly polar water molecules strongly interacts with the hydroxyl groups, and hydrogen bonds between polymer chains are weakened. Strong interactions loosen the structure of polymer matrix allowing oxygen molecules to traverse the film structure and thus water molecules are impairing the material barrier performance against oxygen. Simultaneously they are extremely poor water vapour barriers. The transport of gas molecules through materials is not only dependent on chemical interactions and polarity issues but also on the structural features of the material. Thus, porosity, degree of crystallinity, orientation and density strongly affect the gas permeation, water interactions and barrier performance of the polysaccharide based materials. Crystalline regions and orientation within the material structure generate more tortuous pathways for the gas molecules to traverse, thus significantly retarding the permeation rates through the material. ${ }^{12}$ 
Cellulosic materials are also of interest for sensor applications but in contrast to barrier materials these applications require large surface area and a very fast pathway of molecules to passage through the sample to reach desired high sensitivity. The high surface area in combination with robustness of nanocellulosic films has been shown to be advantageous when considering the development of cellulose-based sensors. ${ }^{13}$ In comparison with other microfluidic substrates, cellulose based sensors are less expensive than poly (ethylene terephthalate) and glass based sensors. ${ }^{14,15}$

Supramolecular structure of cellulose is a significant issue when considering the behaviour of cellulosic materials in the presence of water. ${ }^{16}$ Nanoscaled cellulosic materials, especially cellulose nanofibrils (CNFs), form strong and dense films that have shown very good oxygen barrier performance also at elevated humid conditions. ${ }^{3}$ This has been explained by the high crystallinity of the fibrils (degree of crystallinity 40-60\% depending on the fibril source) and high density of the films. ${ }^{17,18}$ However, the effect of cellulose crystallinity on interactions with water has not been discussed in detail. Furthermore, the influence of porosity on film strength has been determined ${ }^{19}$ but film porosity has not been directly correlated with permeability or with molecular interactions in the presence of water.

In this context, it is crucial to understand the response of thin nanoscaled cellulosic films and the effect of their supramolecular structure (porosity, roughness and crystallinity) towards changes in surrounding conditions, especially when exposed to different humidity levels and when directly immersed in water. Thin welldefined cellulosic films have been extensively studied using surface sensitive techniques like atomic force microscopy (AFM) or quartz crystal microbalance with dissipation monitoring (QCM-D) ${ }^{20}$ and it has been previously shown that structure and chemical composition of the cellulose film indeed affect the swelling behaviour in aqueous media. ${ }^{21}$ However the cellulosic substrates used in these investigations were prepared from varying sources of cellulose (e.g. cellulose nanofibrils from spruce, cellulose derivative and dissolved cellulose) and the distinct effect of crystallinity and/or porosity for chemically identical thin cellulose films still remains unknown. Furthermore, attempts to investigate the water vapour uptake ability of cellulosic materials have been carried out mainly using bulk methods such as Dynamic Water Sorption (DVS) analysis ${ }^{22-}$ ${ }^{24}$ whereas with the QCM-D method, which is equipped with humidity module, not only the water uptake and release of the cellulose thin films can be systematically revealed but also the changes in viscoelastic properties (film softness or rigidity) when exposed to different levels of relative humidity can be clarified. ${ }^{26-27}$ These changes can be linked to the performance of lignocellulosic films and materials in order to gain deeper understanding on their water interactions.

The overall aim of the present study is to obtain deeper understanding on the water interactions of plant derived nanoscaled materials and structures. In this work the direct assessment of water interactions of cellulosic materials with different supramolecular structures are systematically carried out using a set of surface sensitive methods. Regenerated cellulose thin films with identical chemical composition and simultaneously with different degree of order (crystallinity) are prepared by means of spin coating and Langmuir-Schaefer deposition resulting in highly amorphous and more crystalline cellulose films, respectively. The water vapour uptake and swelling behaviour, and water induced changes in morphology are analysed using QCM-D and AFM, and the findings are interpreted with respect to thin film morphology. Furthermore, the cellulosic structures are surface modified via adsorption by using etherified xylan derivative with hydrophobic moieties, (1butyloxy-2-hydroxyl-propyl xylan), in order to investigate such simple strategy to tailor the water interactions towards the desired direction and finally, the changes in moisture uptake ability subsequent to xylan derivative adsorption were investigated.

\section{Materials and methods}

Preparation of trimethylsilyl cellulose (TMSC). TMSC was synthesized by silylation of microcrystalline cellulose powder from spruce (Fluka) with hexamethyldisilazane (Fluka) as previously described. ${ }^{28}$ The degree of substitution was determined to be 1.6 by XPS according to a method described earlier. 28,29

Preparation of 1-butyloxy-2-hydroxy-propyl xylan. Xylan extracted from bleached kraft pulp (Betula Pendula) was etherified according to procedures reported earlier. ${ }^{30}$ The molar ratio of butyl glycidyl ether/anhydroxylose was 2.0. The dialyzed sample was freeze-dried yielding 1-butyloxy-2-hydroxy-propyl xylan, see chemical structure in Figure 1.13C NMR spectra were recorded using a Bruker Avance III $500 \mathrm{MHz}$ spectrometer equipped with a z-gradient double-resonance probe ${ }^{30}$ and the degree of substitution of 0.7 was determined.

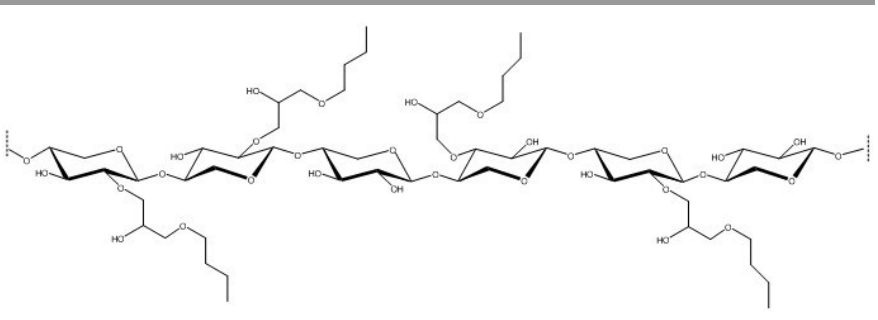

Figure 1. Chemical structure of 1-butyloxy-2-hydroxy-propyl xylan with the degree of substitution of 0.7 .

All chemicals were of analytical grade if not otherwise indicated and the water used was deionized and further purified using Millipore Synergy UV unit with $0.22 \mu \mathrm{m}$ membrane. Milli-Qwater (resistivity $18.2 \mathrm{M} \Omega$ ) was used throughout the study.

QCM-D sensors. The sensors crystals were AT-cut quartz crystals supplied by Q-sense AB, Gothenburg, Sweden. The fundamental resonance frequency, $f_{o}$ was $5 \mathrm{MHZ}$ and the sensitivity constant $\mathrm{C} \approx 0.177 \mathrm{mg} \mathrm{m}^{-2} \mathrm{~Hz}^{-1}$. Prior to cellulose deposition, the gold crystals were first cleaned by UV/ozone treatment for $30 \mathrm{~min}$ followed by spin coating with $2 \mathrm{wt}$ \% polystyrene in toluene $(4000 \mathrm{rpm}, 60 \mathrm{sec})$. The coated crystals 
were dried in an oven at $60^{\circ} \mathrm{C}$ for at least $10 \mathrm{~min}$ in order to ensure sufficient adhesion between base substrate and the first TMSC layer.

Preparation of highly amorphous cellulose thin film. Highly amorphous cellulose substrates were prepared by spin coating TMSC onto polystyrene coated QCM-D crystals using slightly modified previously reported procedure. ${ }^{31} 40 \mu \mathrm{L}$ of TMSC in chloroform solution $(18.5 \mathrm{mM})$ was applied to the substrate and the sample was spinned at $4000 \mathrm{rpm}$ with the acceleration speed of $2130 \mathrm{rpm} / \mathrm{sec}$ for 60 seconds to obtain thin cellulose films with the areal mass of $39 \pm 8 \mathrm{mg} \mathrm{m}^{-2}$ (thickness of $33 \pm 7 \mathrm{~nm}$ ) after desilylation. These substrates are in the following denoted SC or highly amorphous cellulose films.

Preparation of more crystalline cellulose thin film. The horizontal Langmuir-Schaefer (LS)-deposition method was used to prepare more crystalline cellulose substrates. ${ }^{28}$ About 60 layers of TMSC were deposited on the previously prepared polystyrene coated gold crystal at a constant surface pressure of $15 \mathrm{mN} \mathrm{m}^{-1}$ to obtain thin cellulose films with the areal mass of $39 \pm 8 \mathrm{mg} \mathrm{m}^{-2}$ (thickness of $33 \pm 7 \mathrm{~nm}$ ) after desilylation. These films are called LS films or more crystalline films.

Both types of TMSC films (LS and SC) were converted to cellulose via desilylation in humid $\mathrm{HCl}(\mathrm{g})$ atmosphere for 2 min. ${ }^{32,} 33$ These thin films were very sensitive to contamination and variation in relative humidity in the air and thus the desilylated films were stored in a dark desiccator for at least 48 hours, but not more than 100 hours prior to water vapour uptake measurements, to ensure repeatable results.

Atomic Force Microscopy (AFM). AFM was used to characterize the coverage, morphology, and roughness of the thin cellulose films before and after adsorption of 1-butyloxy-2hydroxy-propyl xylan. The samples were imaged in tapping mode in air at room temperature $\left(23^{\circ} \mathrm{C}\right)$ using Multimode AFM with a Nanoscope $\mathrm{V}$ controller (Bruker Corporation, Massachusetts, USA). Silicon cantilevers (NSC15/AIBS), fundamental resonance frequency of about $320-370 \mathrm{kHz}$ and a nominal spring constant of $40 \mathrm{~N} \mathrm{~m}^{-1}$ delivered by MikroMasch (Tallinn, Estonia) with a tip radius below $10 \mathrm{~nm}$ were used. At least there different areas on each sample were analyzed and no image processing except flattening was performed. The RMS (root-mean-square) roughness and skewness were determined from the $1 \mu \mathrm{m}^{2}$ height images with the aid of the Nanoscope Analysis 8.15 software from Bruker Corporation.

Quartz Crystal Microbalance with Dissipation monitoring (QCM-D). The water-uptake/release and water induced swelling of SC and LS cellulose films as well as the adsorption of 1butyloxy-2-hydroxy-propyl xylan on cellulose were studied using the E4 instrument from Q-Sense AB, Götenborg, Sweden. The QCM-D technique enables the in situ studies of mass changes taking place at solid/liquid and solid/gas interface. The piezoelectric quartz crystal oscillates at a resonance frequency fo which is lowered or increased when mass changes are sensed on the surface of the crystal. The interpretation of the QCM-D data is described in detailed elsewhere. ${ }^{34,} 35$ If the layer is evenly distributed, rigidly attached, fully elastic and small compared to the mass of the crystal, the change in frequency is directly proportional to the mass change per unit surface $(\Delta \mathrm{m})$, by the Sauerbrey equation. ${ }^{36}$

$$
\Delta m=-C \frac{\Delta f}{n}
$$

where $\Delta f=f-f_{0}$ is the resonance frequency, $n$ is the overtone number $(n=1,3,5,7, \ldots)$ and $C$ is a constant that describes the sensitivity of the device to changes in mass. For the crystals used $C \approx 0.177 \mathrm{mg} \mathrm{m}^{-2} \mathrm{~Hz}^{-1}$

The resonant frequency of the crystal depends on the total oscillating mass, including the water coupled to the oscillation. By measuring simultaneously several overtones of frequency and dissipation responses, it becomes possible to determine whether the layer on the sensor surface is fully elastic or viscoelastic which is not possible by looking only at the frequency response. If the film on the sensor surface is not fully elastic (rigidly adhered), frictional losses occur that lead to damping of the oscillation with a decay rate of amplitude which is dependent on the viscoelastic properties of the material. With the QCM-D the change in dissipation $\left(\triangle D=D-D_{0}\right)$ is measured and the energy dissipation, $D$, is defined by

$$
D=\frac{E_{\text {diss }}}{2 \pi E_{\text {stor }}}
$$

where $E_{\text {diss }}$ is the total dissipated energy during one oscillation cycle, $E_{\text {stor }}$ is the total energy stored in the oscillation. The change in dissipation $(\triangle D)$ measures qualitatively the rigidity and softness of the film adhered on the sensor surface. The film can be considered fully elastic and rigid when $\Delta D \leq 1 \times 10^{-6}$ and there is no spreading of the overtones.

Film thickness determination by QCM-D. The thickness of the SC and LS-deposited cellulose thin films was determined using a QHM 401 humidity module coupled with the QCM-D. ${ }^{26}$ The thickness of the cellulose model surfaces was calculated based on the difference in frequency response before and after film deposition measured in air. Changes in frequency were monitored before and after cellulose film deposition in air upon stabilization of the signal at $23{ }^{\circ} \mathrm{C}$ with an air flow of $0.1 \mathrm{ml} \mathrm{min}-$ ${ }^{1}$ for $25 \mathrm{~min}$. Collected frequency data was stiched together by QTools software and finally the thickness of the films was calculated according to Eq. 3 assuming the layer density of 1200 $\mathrm{kg} \mathrm{m}^{-3}$ for the deposited thin cellulose film.

$$
h=\frac{\Delta m_{\text {Sauerbrey }}}{\rho_{\text {Assumed }}}
$$

Water vapor uptake and release determination by QCM-D. The QHM 401 humidity module was used to study the water vapour uptake and release and the effect of relative humidity on viscoelastic properties of the cellulose thin films. Prior to the water vapour uptake and release measurements the cellulose 
films were allowed to stabilize inside the module under the exposure to the relative humidity level of $11 \%$ for $40 \mathrm{~min}$. Changes in frequency and dissipation were monitored as a function of time with gradually increasing relative humidity (RH) by circulating different saturated salt solutions (Table 1.) through the humidity module with the flow rate of $0.1 \mathrm{ml} \mathrm{min} \mathrm{m}^{-1}$ at $23^{\circ} \mathrm{C}$ for $30 \mathrm{~min}$.

Table 1. Different saturated salt solutions and respective relative humidity levels used for water vapour uptake and release measurements monitored with QCM-D. ${ }^{37}$

\begin{tabular}{cc}
\hline Saturated salt solution & Relative humidity $(\%)$ at $23{ }^{\circ} \mathrm{C}$ \\
\hline $\mathrm{LiCl}$ & 11 \\
$\mathrm{MgCl}_{2}$ & 33 \\
$\mathrm{Mg}\left(\mathrm{NO}_{3}\right)_{2}$ & 53 \\
$\mathrm{NaCl}$ & 75 \\
$\mathrm{~K}_{2} \mathrm{SO}_{4}$ & 97 \\
$\operatorname{milliQ~}_{2} \mathrm{O}$ & 100 \\
\hline
\end{tabular}

Adsorption measurements using QCM-D. For the adsorption experiments the aqueous 1-butyloxy-2-hydroxypropyl xylan solution was prepared followed by overnight stirring at room temperature $23{ }^{\circ} \mathrm{C}$. The solution was filtered through $0.45 \mu \mathrm{m}$ membrane before use. Prior to adsorption investigations the cellulose surfaces were allowed to stabilise in milliQ water to ensure stable zero baselines for the frequency and dissipation changes. After replacement of water in the QCM-D chamber with a solution containing $100 \mathrm{mg} \mathrm{l}^{-1}$ xylan derivative, frequency and dissipation changes were recorded as a function of time for $140 \mathrm{~min}$ with the flow rate of $0.1 \mu 1 \mathrm{~min}^{-1}$ with subsequent rinsing with pure milli-Q water. Each measurement was repeated at least twice.

\section{Results and discussion}

\section{Structural features of cellulose thin films}

In order to better understand how the supramolecular structure of cellulose (crystallinity, roughness and porosity) is affecting the interactions with water, the cellulose thin film approach with surface sensitive methods (QCM-D and AFM) was applied. By this approach the well-defined cellulose substrates with identical chemical composition simultaneously with the different degrees of order can be provided. Thus, the cellulose thin film surfaces utilised here provide not only the defined chemistry but also the controlled morphology and thickness which enables the systematic comparison of the water uptake ability of physically and structurally different cellulose materials. For this purpose cellulose thin films of equal thicknesses with different degrees of order were prepared using Langmuir-Schaefer (LS) technique and spin coating (SC) technique. The film thickness values were calculated through the changes in frequency response before and after the deposition of the cellulose layer on the QCM-D sensor surface by applying the Equations 1 and 3, (see also Figure 2).

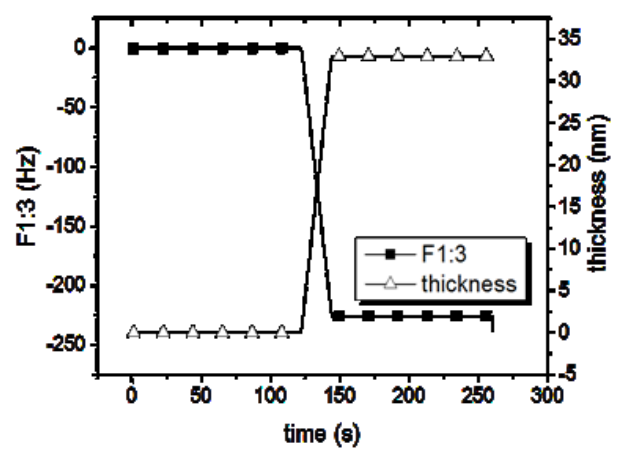

Figure 2. Example of the cellulose film thickness determination through the frequency response and calculated using Sauerbrey equation.

The film thickness calculated by Equations 1 and 3 assuming that the density of the formed cellulose layer deposited on the sensor surface is $1200 \mathrm{~kg} \mathrm{dm}^{-3}$ yields the thickness of approximately 33 $\mathrm{nm}$. Care was taken that both deposition methods always yielded the thickness values of $\sim 33 \mathrm{~nm}$, and the thickness variation never exceeded $7 \mathrm{~nm}$ as formerly reported as acceptable and attainable thickness variation for this particular surface analysis approach. ${ }^{26}$ Previous Small Incidence Angle X-ray Diffraction (SAXS) measurements have shown that LS films exhibit a degree of crystallinity of about $60 \%$ and the crystal structure is cellulose II, as expected for a regenerated cellulose film. ${ }^{21}$ On the other hand the herein exploited spin coating procedure generates highly amorphous and unoriented regenerated cellulose films. ${ }^{38,} 39$ Therefore, chemically identical pure cellulose films with different structural features were obtained, and the influence of crystallinity, surface roughness and film porosity on the water interactions at molecular level can be systematically and directly investigated with nanoscale sensitivity.

The effect of film formation method on morphology and surface roughness of the cellulose films was determined using AFM. In Figure 3, AFM height images and surface profiles of regenerated Langmuir Schaefer (LS) deposited cellulose film after 60 dipping cycles and spin coated (SC) cellulose film are shown. From the surface profiles and surface roughness values it is clear that the LS cellulose film is smoother compared to the SC film. The RMS roughness for $1 \mu \mathrm{m}^{2}$ area is 0.5 and $3.4 \mathrm{~nm}$ for LS and $\mathrm{SC}$ films respectively. This is as expected since in LS-deposition one bi-layer is deposited onto the polystyrene-coated gold crystal during each dipping cycle, and as a consequence the layer thickness can be controlled without changing the roughness of the film. In spin coating the thickness and roughness is dependent on the concentration of the TMSC solution. To obtain thick enough films rather concentrated solutions had to be used and therefore slightly rougher films were obtained as compared to previously reported thinner films. ${ }^{31}$ 
A detailed study of the LS film topography reveals that the film had a layered structure and one monolayer was not fully covering the substrate. This introduced a nanoscaled porosity that influenced the interaction with water as will be demonstrated in the following sections. Surface skewness $\left(\mathrm{S}_{\mathrm{sk}}\right)$ values which describe the asymmetry of the height distribution were measured from AFM data. ${ }^{40}$ Surface skewness of LS films showed negative values $(-0.2 \mathrm{~nm})$ indicating that the LS sample has porous surface, whereas in case of spin coated films the surface skewness values were positive $(0.7 \mathrm{~nm})$, indicating that there are more peaks than valleys or pores. The spin coated film is fully covering the substrate already with one layer and the surface features are larger, thus, even though the roughness is higher for the SC sample, the available surface area is smaller.

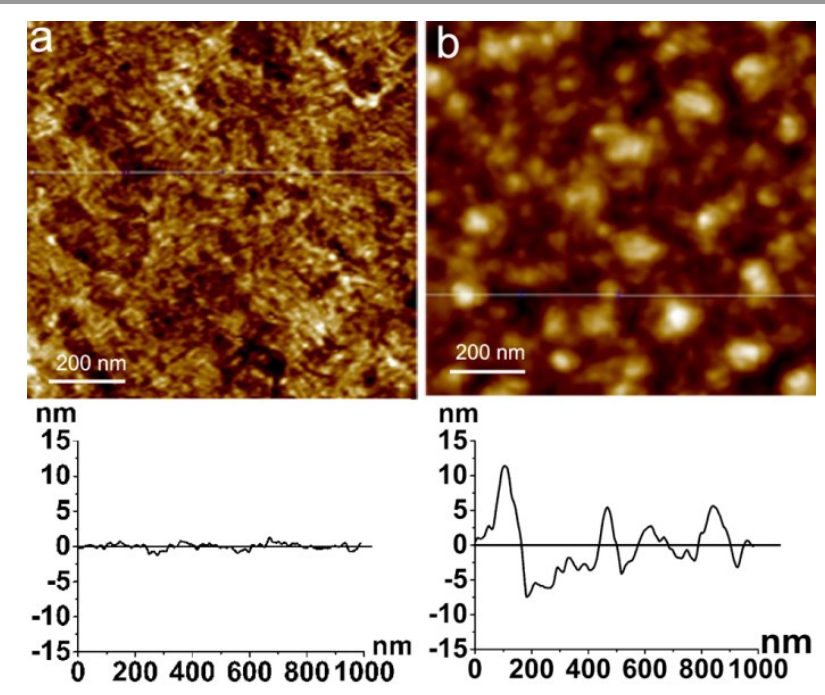

Figure 3. AFM images and corresponding surface profiles for (a) more crystalline cellulose film, (b) highly amorphous cellulose film on polystyrene coated gold substrates. The scan size is $1 \mu \mathrm{m}^{2}$ and the z-range is $15 \mathrm{~nm}$.

\section{Cellulose thin film interactions with water molecules}

Water vapour uptake and softening of cellulose thin films. The systematic comparison of the water uptake and release ability of physically and structurally different cellulose materials i.e. highly amorphous and more crystalline cellulose films were carried out using QCM-D equipped with the humidity module. Mass changes due to water uptake and release as well as concomitantly detected water induced rheological changes in the cellulose thin film behaviour were detected by following the change in frequency and change in dissipation as a function of gradually increasing/decreasing relative humidity.

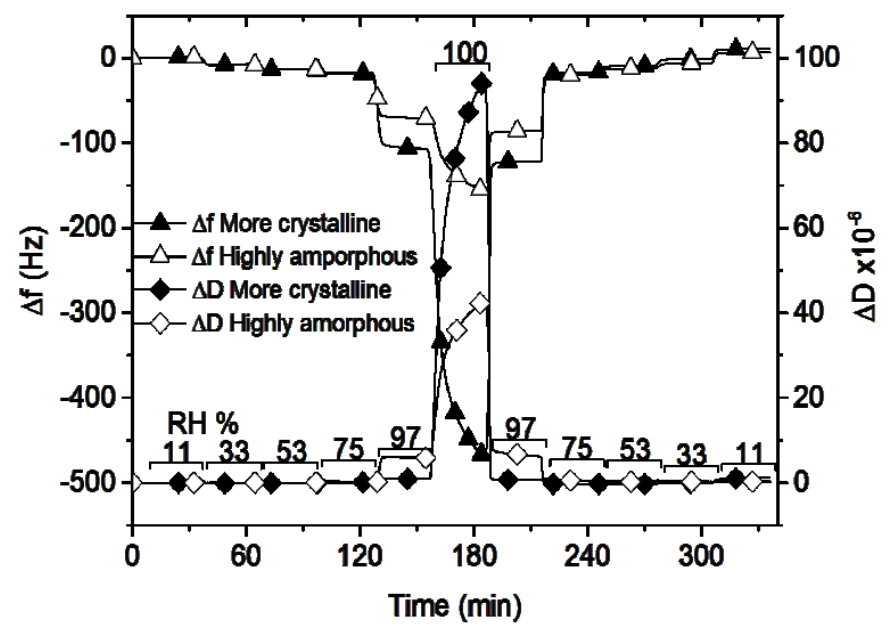

Figure 4. Change in frequency (triangles) and dissipation (diamonds) as a function of time for highly amorphous (open marks) and more crystalline (filled marks) cellulose films at different levels of humidity. $\left(f_{0}=5 \mathrm{MHz}, n=3, f_{3} / n\right)$.

Figure 4 shows an example of the humidity uptake/release QCMD measurement for both highly amorphous SC cellulose film and more crystalline LS cellulose film. The negative frequency change followed as a function of increasing relative humidity and as a function of time indicates the mass uptake detected by the sensor due to the water vapour uptake. The positive frequency change is related to the release of water molecules from the cellulose surface. Simultaneously recorded dissipation changes give indication on the rheological properties of the cellulose film under the exposure to different relative humidity. Increase in dissipation indicates the formation of soft viscoelastic film, whereas negligible changes in dissipation indicate rigid and fully elastic film. The first humidity step in Figure 4 (RH 11\%) is associated with the stabilisation step of the entire humidity measurement and therefore it is set as a baseline in which no changes in frequency or dissipation are detected, and the cellulose films are in the equilibrium state with the surrounding humidity. After the equilibrium step the relative humidity inside the measurement cell was gradually increased up to $\mathrm{RH} 100 \%$ and each humid conditions were maintained approximately 30 min. Up to RH $75 \%$ both cellulose surfaces were capable to adsorb similar amount of water molecules whereas the dissipation change remained zero. This indicates that both more crystalline and highly amorphous cellulose films remained rigidly attached on the sensor surface although water molecules were interacting with the film. When the humidity reached the RH 97\% clear changes took place when considering the mass uptake and finally at RH $100 \%$ the water uptake was even more pronounced. Due to the water condensation taking place at $\mathrm{RH}$ $100 \%$, the real equilibrium cannot be reached, and therefore the most reliable responses to be compared are those collected at humidity values lower than $100 \%$. Changes in frequency and dissipation showed the similar response towards gradually decreasing humidity level in the reverse order. Finally when reaching the $\mathrm{RH}$ level of $11 \%$, frequency change values for both cellulose layers are slightly higher compared to the original 
baseline level values. This is probably due to the fact that the utilized stabilisation time (40 min prior to the measurement at RH $11 \%$ ) is somewhat inadequate when considering such hygroscopic material as cellulose.

Interestingly, the more crystalline LS cellulose film seems to be capable to uptake higher amount of water compared to the highly amorphous cellulose layer at high humidity (RH 97\%). This is surprising since tightly packed crystalline regions are less permeable to water. On the other hand, at the same time the $\Delta \mathrm{D}$ is increasing more for the amorphous film as compared to the LS film. In order to reveal more thorough information on the behaviour of cellulosic material in the presence of water molecules and to better explain the aforementioned finding, the QCM-D data is presented in a more detailed manner in Figure 5. Only the most reliable part $(\mathrm{RH}<100 \%)$ of the water vapour uptake is compared, and the frequency as well as dissipation responses which are recorded using several overtones $(n=1,3$, $5,7,9)$ are shown. By using data of several overtones the viscoelastic behaviour can be interpret comprehensively since e.g. spreading of the frequency and dissipation curves recorded at different overtones is an indication of the formation of viscoelastic layers instead of fully elastic layer. Water uptake data calculated as areal mass increase determined using Sauerbrey equation, and as milligrams of water bound by milligram of cellulose are both summarized in Table 2. As already mentioned, the more crystalline cellulose thin film was capable to uptake more water as compared to highly amorphous cellulose thin film especially when exposed to high level of relative humidity ( $\mathrm{RH} 97 \%$ ). Approximately $33 \mathrm{~nm}$ thick more ordered layer could absorb $\sim 0.5 \mathrm{mg}$ water whereas the value was $\sim 0.3 \mathrm{mg}$ for highly amorphous film with the similar thickness, see Table 2. The reason for the larger water uptake ability may originate from the differences in structural features of SC and LS cellulose films.
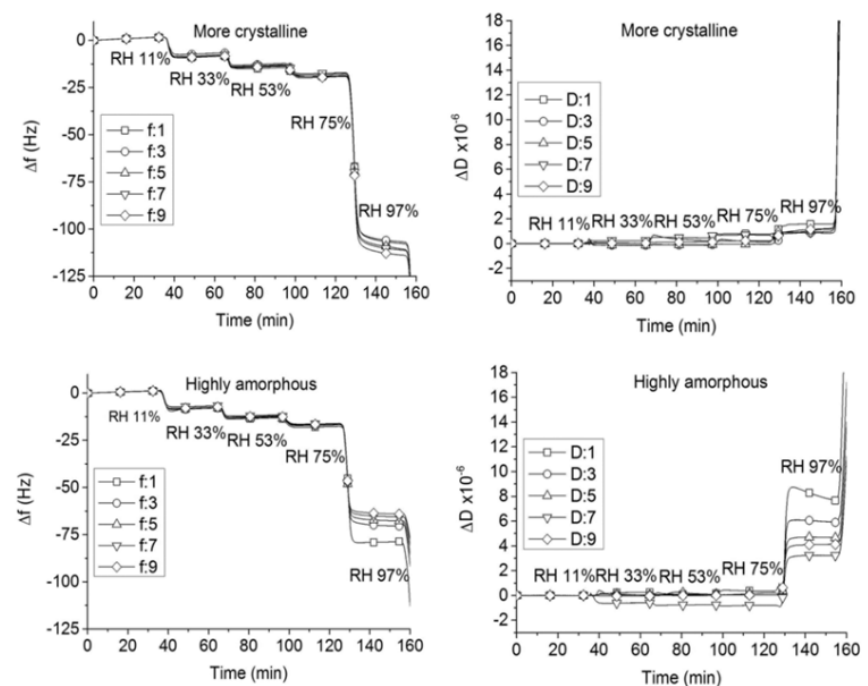

Figure 5. Top row: spreading of the overtones for more crystalline cellulose thin film: frequency change (left) and dissipation change (right). Bottom row: spreading of overtones for highly amorphous cellulose: frequency change (left) and dissipation change (right)
As revealed by AFM analysis, the more crystalline LS cellulose surface had a layered structure with nanoscaled porosity. This structural feature has also been previously reported for cellulose thin films with significant crystallinity. ${ }^{28,} 41$ This feature is expected to influence on the interactions with water since the porous layer may contain more available surface hydroxyl groups due to the higher specific surface area compared the amorphous cellulose surface. Therefore, the more crystalline cellulose layer with the similar thickness and identical chemistry can uptake more water molecules compared to fully and evenly covering non-porous amorphous SC cellulose film.

Concomitantly, the dissipation change at $\mathrm{RH}$ of $97 \%$ was significantly higher for amorphous film although the amount of bound water was lower; compare the Figure $5 \mathrm{~b}$ and $5 \mathrm{~d}$. The change in dissipation is approximately $1 \times 10^{-6}$ for more crystalline cellulose and as high as $6 \times 10^{-6}$ for highly amorphous cellulose (values measured using the $3^{\text {rd }}$ overtone). This can be interpreted in terms of softness and rigidity of the films: more crystalline cellulose film remained rigid although the hydroxyl groups can effectively bind water molecules, whereas lower amount of bound water induced cellulose layer softening of the highly amorphous cellulose structure. Furthermore, as shown in Figure 5, both frequency and dissipation curves appears to spread more extensively in the case of the highly amorphous SC cellulose substrate indicating more severe changes in layer's rheological behaviour as a function of relative humidity i.e. fully elastic layer becomes viscoelastic at high humid conditions.

Several water uptake mechanisms can be expected to be involved when considering water interactions with highly hydrophilic cellulose surfaces. Hydrophilic polymers such as cellulose and many other biobased materials typically display sigmoidal shaped water vapour adsorption isotherms due to stronger moisture uptake at very low or very high relative humidity. ${ }^{42}$ This behaviour can be linked to fast and slow kinetic processes related to fast and slow sorption sites for water molecules to interact with hydrophilic structures. In literature the sigmoid curve is further interpreted by models based on Langmuir monolayer adsorption, BET (Brunauer, Emmet and Teller) multilayer adsorption, D'Arcy Watt equation, Flory-Huggins mixing theories and PEK-model (Parallel Exponential Kinetic model). ${ }^{23}$, 43-48 These thorough moisture sorption investigations have been performed on bulk materials mainly using dynamic water vapour sorption methods and below we utilize this knowledge to further elaborate on the water uptake mechanisms taking place in our system, which exploits surface sensitive methods and ultrathin films of cellulose. At low relative humidity individual water molecules strongly bind to cellulose surface hydroxyl groups finally leading to the formation of a monolayer of water. In the slow kinetics region $(11 \%<\mathrm{RH}<97 \%)$ water molecules can be expected to have occupied most of the reactive sites, see Figure 5 and Table 2. Slow sorption process has been explained as indirect sorption onto the internal cellulose surface. Simultaneously the adsorbed moisture may induce polymer swelling and rearrangements of the cellulose molecules. ${ }^{47}$ It has also been suggested that water clusters are formed during the slow sorption region, i.e. additional water 
molecules are adsorbed on the water molecules already bound to the cellulose surface. ${ }^{43,} 47$ In our system significant layer softening was not detected at humid conditions lower than RH97\%, indicating that moisture induced swelling and clear molecular arrangements did not take place below this humidity, not even in the case of highly amorphous cellulose. In addition, both the highly amorphous and the more crystalline films were capable to uptake similar amount of water molecules, which suggests that the water uptake mechanism was identical up to this point. Adsorption models based on rate theory only, such as Langmuir and BET, seem to be applicable until rather high relative humid conditions, since no indication on layer softening is observed. At high relative humidity, in the region of fast sorption kinetics, water condensation and polymer solvation can take place. Based on D'Arcy Watt's assumption, water interacts with sites where water condenses as a collection of molecules. For soft polymers, which strongly interact with water and for mounted in the QCM-D chamber and the measurements were started in air, and at $\mathrm{t}=0$ milli-Q-water was injected. The injection of water caused a sudden drop in frequency and an increase in in energy dissipation because of the viscosity difference between air and water and also due to the rapid water uptake of the cellulose substrates. The changes in frequency and dissipation caused by changing the media were also monitored for a pure gold crystal to determine the bulk effect. The following values were achieved for the third overtone: $\Delta f=-386 \mathrm{~Hz}$ and $\Delta \mathrm{D}=165 \times 10-6$. Consequently the remaining change in frequency $(\Delta f)$ can be attributed to the swelling of the cellulose films.

Table 2. Water vapour uptake data for the highly amorphous and more crystalline cellulose layers. Average and deviation of two measurements.
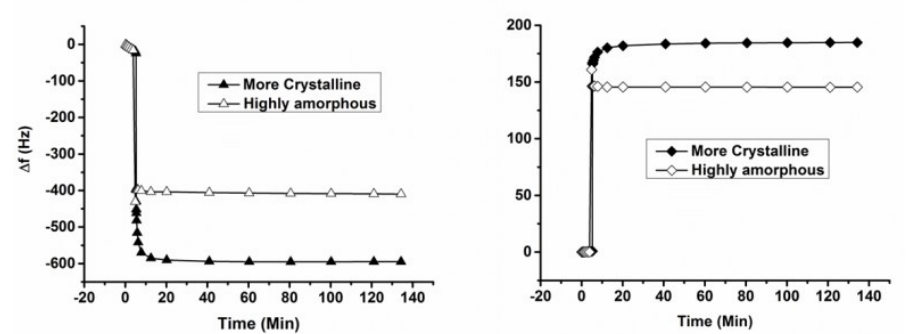

which swelling and molecular rearrangements may take place, the Flory-Huggins mixing theory can be applied as well. ${ }^{44}$ As can be seen from the data both the cellulose films swell and thus, they strongly interact with water. The thermodynamic equilibrium is a result of interaction energy between polymer chains and water, and the entropy term of mixing and the mechanism seems to be related to water solvation. However, the more crystalline cellulose, having nanoporous structure, was capable to uptake more water based on direct mass calculations (see Table 2) and the swelling was significantly less pronounced. The other mechanism taking place here may be related to capillary condensation inside the nanopores and a larger surface area. Water molecule droplets adsorbed on the pore surfaces are condensed to thin water films within the pores at high RH levels, seen as higher water vapour uptake ability. Nevertheless, the water cannot penetrate inside crystalline parts of the substrate. Altogether, the chemical composition of the two cellulose surfaces is identical and therefore it seems that the differences in water vapour uptake ability and swelling in this study cannot be interpreted in terms of chemical composition and hydrophobic interactions as has been previously reported..$^{23}$

Swelling of cellulose films in water. The investigation of cellulose interaction with water was continued further to evaluate the swelling of the cellulose substrates upon immersion in water. Figure 6 shows the swelling behaviour of more crystalline LS cellulose substrate and highly amorphous spin coated cellulose film. In contrast to previous studies ${ }^{20}$ here the films of equal thickness $(33 \mathrm{~nm})$ were compared. Dry cellulose films were

\begin{tabular}{|c|c|c|c|c|}
\hline \multirow[b]{2}{*}{$\begin{array}{l}\mathrm{RH} \\
(\%)\end{array}$} & \multicolumn{2}{|c|}{$\begin{array}{c}\text { Highly amorphous cellulose } \\
\text { film (SC) }\end{array}$} & \multicolumn{2}{|c|}{$\begin{array}{c}\text { More crystalline cellulose } \\
\text { film (LS) }\end{array}$} \\
\hline & $\begin{array}{c}\text { Areal mass } \\
\left(\mathrm{mg} \mathrm{m}^{-2}\right)\end{array}$ & $\begin{array}{l}\mathrm{mg} \mathrm{H}_{2} \mathrm{O} / \mathrm{mg} \\
\text { cellulose }\end{array}$ & $\begin{array}{c}\text { Areal mass } \\
\left(\mathrm{mg} \mathrm{m}^{-2}\right)\end{array}$ & $\begin{array}{c}\mathrm{mg} \mathrm{H}_{2} \mathrm{O} / \mathrm{mg} \\
\text { cellulose }\end{array}$ \\
\hline 11 & \multicolumn{2}{|c|}{$\begin{array}{l}\text { Stabilization of } 33 \mathrm{~nm} \text { cellulose } \\
\text { surface }\left(39.6 \mathrm{mg} \mathrm{m}^{-2}\right)\end{array}$} & \multicolumn{2}{|c|}{$\begin{array}{c}\text { Stabilization of } 32 \mathrm{~nm} \text { cellulose } \\
\text { surface }\left(38.4 \mathrm{mg} \mathrm{m}^{-2}\right)\end{array}$} \\
\hline 33 & 41.0 & $0.035 \pm 0.001$ & 38.5 & $0.032 \pm 0.002$ \\
\hline 53 & 41.9 & $0.057 \pm 0.001$ & 40.6 & $0.056 \pm 0.003$ \\
\hline 75 & 42.6 & $0.077 \pm 0.002$ & 41.6 & $0.080 \pm 0.004$ \\
\hline 97 & 48.0 & $0.334 \pm 0.026$ & 57.1 & $0.458 \pm 0.033$ \\
\hline
\end{tabular}

Figure 6. Change in frequency (left) and change in dissipation (right) as a function of time upon exposure to water for more crystalline and highly amorphous

Figure 6 show that, most of the water uptake occurred in the initial phase, which is observed as a steep decrease in the frequency and increase in dissipation whereupon $\Delta f$ and $\Delta D$ leveled off. The decrease in frequency $(\Delta f)$ indicates binding of water to the film and changes in dissipation $(\Delta D)$ describes changes in the structural properties such as layer softening and thickening upon water adsorption. Similarly to the uptake of water vapour, the more crystalline LS cellulose adsorbed more water than the spin coated highly amorphous substrate. This is again due to the higher surface area of the LS substrate due to porous and layered structure. The $\Delta D$ curve follows the trend of the frequency shift and is closely related to the effect of the adsorbed water on viscoelastic properties of the swollen layer. The final degree of swelling (mass of water/ dry mass of the film) was calculated to be $72 \%$ for the more crystalline LS film and cellulose. $\left(f_{0}=5 \mathrm{MHz}, n=3, f_{3} / n\right)$. 
$4.8 \%$ for the highly amorphous film. It is difficult to compare these results to previous findings on swelling of cellulose model films, since this is the first time both chemistry and thickness is controlled. Qualitatively the results were similar to previous finding ${ }^{21}$ but due to differences in thickness, the calculated swelling values were quite different. In accordance with Aulin et $a l .{ }^{21}$ it was observed that the LS film swells slowly, seen as the gradual decrease in frequency and increase in dissipation that levelled off after about 20 minutes. In contrast, the amorphous SC cellulose substrate immediately reaches a plateau value. This difference in behaviour correlates well with the higher degree of order and observed higher rigidity of the LS film in high humidity (Figure 5). The higher rigidity leads to slower swelling.

\section{Modification of the cellulose surface via adsorption of etherified xylan derivative}

1-butyloxy-2-hydroxy propyl xylan is a xylan derivative with a more hydrophobic part introduced to its structure, see Figure 1. In the previous study the similar xylan derivative has shown rather interesting conformational rearrangements resulting in higher hydrophobicity when exposed to different humid conditions and temperatures. ${ }^{26}$ Thus, it can be expected to have influence on moisture sensitivity of polysaccharidic materials posited that the degree of substitution of the more hydrophobic moiety is high enough. ${ }^{26}$ Moreover, the properties of cellulose thin films can be efficiently modified by polymer adsorption and many water-soluble renewable polysaccharides have evidenced affinity towards cellulose. ${ }^{49}$ Thus, they can be considered as attractive choices for this purpose. Surface modification strategy via adsorption is performed in aqueous media and is thus environmentally friendlier and easier compared e.g. to silylations or other methods performed in organic solvents.

In Figure 7 the areal mass changes $(\Delta m)$ and change in dissipation $(\Delta D)$ upon adsorption of the 1-butyloxy 2-hydroxy propyl xylan with the degree of substitution of 0.7 on the two morphologically different cellulose substrates are shown. From the figure it is evident that the 1-butyloxy-2-hydroxy propyl xylan adsorbed onto both cellulose surfaces and only a small amount of loosely bound molecules is removed upon rinsing. The areal mass change after rinsing was approximately $5.4 \mathrm{mg}$ $\mathrm{m}^{-2}$ on the more crystalline and $4.4 \mathrm{mg} \mathrm{m}^{-2}$ on the highly amorphous cellulose film as roughly estimated by using the Equation 1. This is slightly higher than previous results for unmodified birch xylan on LS cellulose $\left(3 \mathrm{mg} \mathrm{m}^{-2}\right)^{49}$ and cereal xylan on nanofibrillated cellulose $\left(1.5 \mathrm{mg} \mathrm{m}^{-2}\right)^{50}$ nonetheless showing that the xylan modification did not hinder adsorption at this low degree of substitution. Previously we have also shown that the driving force for affinity and adsorption of birch pulp derived unmodified xylan on cellulose surface is due to the somewhat limited solubility of xylan molecules. ${ }^{49}$ Bleached hardwood xylan forms precipitates already at $\mathrm{pH} 10$ due to the removal of acetyl groups and glucuronic acid groups during pulp bleaching. The xylan derivatisation strategy utilized here, which involves hydroxyalkylation, again improves the solubility of the hardwood derived xylan in water to some extent. ${ }^{30}$ It is interesting to note the difference in kinetics compared to adsorption of unmodified xylan. ${ }^{49}$ The adsorption of the modified xylan was faster, with a very fast initial adsorption during the first ten minutes that levels off after about $50 \mathrm{~min}$. This could be due to the hydrophobic moiety on the modified xylan which may favor the adsorption on cellulose surface. Similar effect was recently found for the adsorption of hydrophobically modified galactoglucomannan on cellulose. ${ }^{51}$
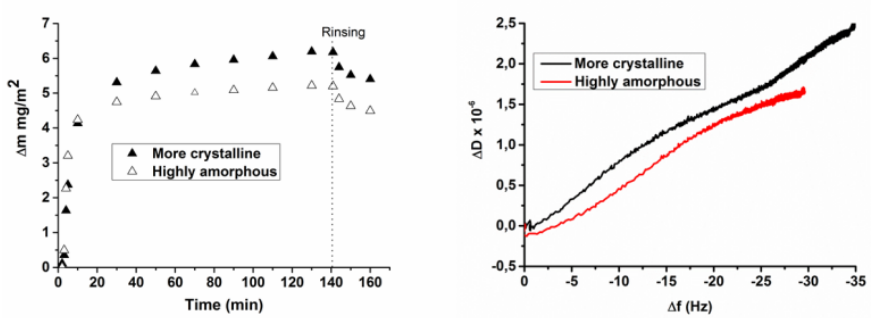

Figure 7. Change in the areal mass $(\Delta \mathrm{m})$ vs. time (left) and change in dissipation $(\Delta \mathrm{D})$ vs. change in frequency $(\Delta f)$ (right) when adsorbing 1-butyloxy 2- hydroxyl propyl xylan on more crystalline and highly amorphous cellulose surfaces. $\left(f_{0}=\right.$ $\left.5 \mathrm{MHz}, n=3, f_{3} / n\right)$

In accordance with previous findings ${ }^{50}$ the adsorbed xylan layer could not be detected using AFM, see Figure 8 . The only change observed in Figure 8 was that the RMS surface roughness of more crystalline film increased considerably from $0.5 \mathrm{~nm}$ to 2.6 $\mathrm{nm}$. A probable explanation for this phenomenon is that some irreversible changes occurred in the supramolecular structure of the film during swelling in aqueous media leading to an increase in the surface roughness. In contrast, no change in surface roughness $(3.4 \mathrm{~nm})$ was observed for the highly amorphous cellulose surface that was already considerably rougher prior to the adsorption experiments. The results indicate that individual xylan derivative molecules are adsorbing on cellulose surface since this xylan derivative is moderately well soluble in water. If the xylan solubility is lower, xylan tends to form small clusterlike assemblies which have been observed on cellulose model substrates. ${ }^{26,49,52}$
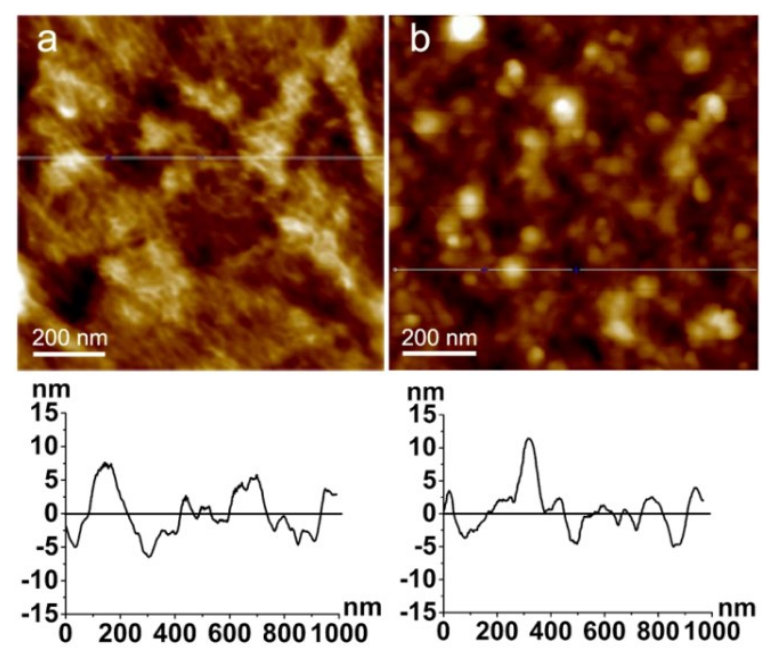

Figure 8. AFM images and corresponding surface profiles for 1-butyloxy-2hydroxyl propyl xylan on (a) more crystalline cellulose and (b) highly amorphous 
cellulose surface after QCM adsorption measurements. The scan size is $1 \mu \mathrm{m}^{2}$ and the z-range is $15 \mathrm{~nm}$.

Finally, the influence of adsorbed layer of etherified xylan derivative on ability of cellulose films to take up water vapour was investigated. Figure 9 shows the humidity uptake for both highly amorphous SC cellulose film and more crystalline LS cellulose film before and after the adsorption of modified xylan. Xylan derivative layer with the areal mass of $5.4 \mathrm{mg} \mathrm{m}^{-2}$ adsorbed on more crystalline cellulose surface did not alter either the humidity response or the viscoelastic behaviour of the film. The film structure was still capable to uptake water molecules but it stayed rigid. In contrast, the behaviour of highly amorphous cellulose substrate with respect to water uptake ability changed upon adsorption of the xylan derivative. $4.4 \mathrm{mg}$ $\mathrm{m}^{-2}$ of adsorbed xylan derivative seems to induce higher water vapour uptake ability at higher humidity conditions.

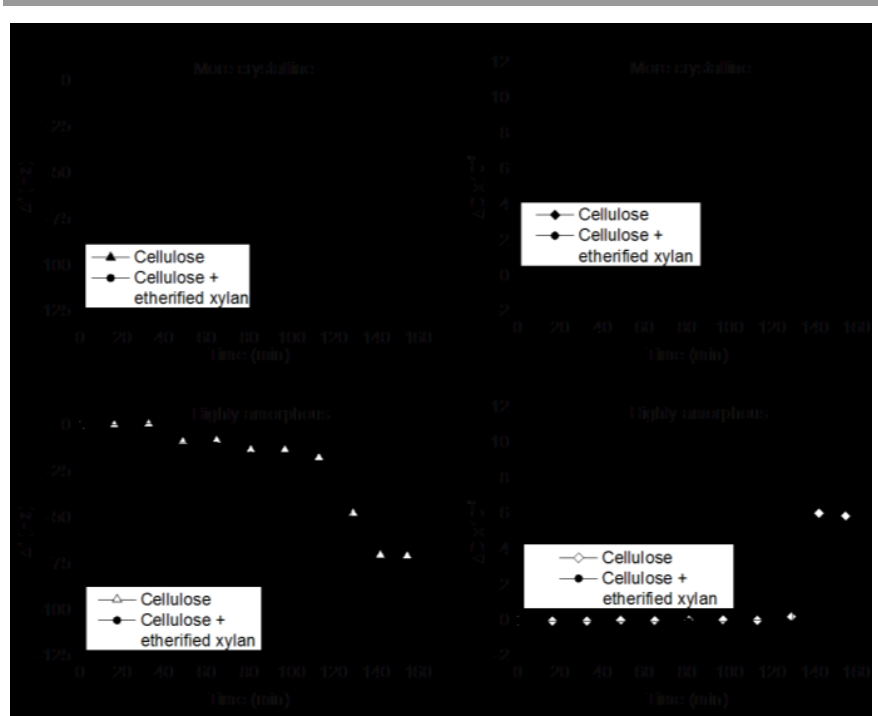

Figure 9. Change in frequency $(\Delta f)$ and dissipation $(\Delta D)$ as a function of time before and after the adsorption of 1-butyloxy-2-hydroxy propyl xylan on cellulose surfaces.

Interestingly, the layer softening was less pronounced, which is seen as lower changes in dissipation values. It seems that cellulose surface morphology has significant influence not only on water interactions, as shown in Figures 4 and 5, but also on the adsorption of polymers. We note that due to the nanoporous structure of the partly crystalline LS cellulose, the xylan was most probably adsorbed inside pores and thus did not affect the interaction with water vapour. In contrast, the amorphous LS cellulose film had a more dense surface (Figure 3 ) and thus it can be assumed that the xylan derivative layer was adsorbed on the top most surface. As a consequence of this and the fact that the degree of substitution of the xylan was quite low, it may generate more sites for water to interact with, leading to higher $\Delta f$. The observed lowered layer softening tendency (i.e. layer is less pronounced to water induced swelling) at high humidity conditions could be due to the hydrophobic parts of the xylan derivative, which can also be expected to be located on the outer most surface. We note that the degree of substitution of the xylan derivative was too low to significantly reduce the interaction with water vapour in either case.

\section{Conclusions}

In this paper direct evidence of the importance of nanostructure of cellulose thin films on surface interactions and on the behaviour of polysaccharide based nanoscaled materials in presence of water molecules was gained. The model cellulose thin film approach was found useful to gain fundamental understanding on the interactions between water molecules and polysaccharides. By controlling the thickness, porosity and crystallinity of chemically identical cellulose thin films it was possible to address the effect of crystallinity and nanoporosity on water sensitivity of cellulose thin films. It was found that a nanoporous but simultaneously partly crystalline cellulose film adsorbed higher amount of water molecules both in humid air and when immersed in water when compared to highly amorphous cellulose substrate probably due to higher surface area generated by the nanopores. Simultaneously such substrates retained their elasticity and rigidity whereas substantial water induced layer softening was taking place when amorphous cellulose substrates were considered. Thin adsorbed layers of 1butyloxy-2-hydroxyl-propyl xylan with a degree of substitution of 0.7 did not alter the water vapour uptake ability of the cellulosic substrates but an interesting correlation between nanostructure of the cellulose and behaviour of the adsorbed xylan was found. The results suggested that the etherified xylan derivative adsorbed on the topmost surface of the dense amorphous cellulose substrate and was, as a consequence, more accessible to water vapour than the nanoporous cellulose substrate. These findings are of high importance when developing strategies to construct efficient, renewable and bioinspired material solutions using nanoscaled building blocks in which for example the gas permeability and moisture sensitivity are relevant aspects. Generalizing these findings it can be concluded that the efficiency of small molecules to affect surface interactions is strongly affected by the substrate morphology. This is a crucial finding especially when considering the applications utilizing ultra thin films and layers such as in sensor elements and in other electronic devices.

\section{Acknowledgments}

Funding for this work was obtained from the Future Biorefinery (FuBio) program financed by TEKES and the Forestcluster Ltd. Finally, the authors would like to acknowledge Ritva Kivelä Elina Niinivaara and Katja Pettersson for their skilful laboratory assistance.

\section{Notes and}

a) VTT Technical Research Centre of Finland, P.O. Box, FI-02044 VTT, Espoo, Finland

b) Aalto University, School of Chemical Technology, Department of Forest Products Technology, P. O. Box 1000, FI- 00076 Aalto, Espoo, Finland 


\section{References}

1 R. A. Gross and B. Kalra, Science, 2002, 297, 803.

2 K.S. Mikkonen and M.Tenkanen, Trends Food Sci. Technol. 2012, 28, 90.

3 N. Lavoine, I. Desloges, A. Dufresne and J. Bras, Carbohydr. Polym., 2012, 90, 735.

4 E. W. Nery and L. T. Kubota, Anal. Bioanal. Chem., 2013, 405 , 7573.

5 M. Nogi and H. Yano, Adv. Mater., 2008, 20, 1849.

6 A. Mautner, K.-Y. Lee, P. Lahtinen, M. Hakalahti, T Tammelin, K. Li and A. Bismarck, Chem. Commun. 2014, DOI: 10.1039/C4CC00467A.

7 N.M. Hansen and D. Plackett, D., Biomacromolecules, 2008, 9, 1493

8 E. Entcheva, H. Bien, L. Yin, C. Chung, M. Farrell and Y. Kostov, Biomaterials, 2004, 25, 5753.

9 K.L. Spence, R.A. Venditti, O.J. Rojas, Y. Habibi and J.J. Pawlak, Cellulose, 2010, 17, 835.

10 S. Ummartyotin, J. Juntaro, M. Sain and H. Manuspiya, Ind. Crop. Prod., 2012, 35, 92

11 O.G. Piringer and A.L. Baner, in Plastic packaging materials for food. Barrier function, mass transport, quality assurance, and legislation, $1^{\text {st }}$ edn. Wiley-VCH, 2000.

12 D.R. Paul and L.M. Robeson, Polymer, 2008, 49, 3187.

13 H. Orelma, I. Filpponen, L. Johansson, M. Österberg, O.J. Rojas and J. Laine, Biointerphases, 2012, 7, 1.

14 S.-C. Tseng, C.-C. Yu, D. Wan, H.-L. Chen, L.A. Wang, M.-C. Wu, W.-F. Su, H.-C. Han and L.-C. Chen, Anal. Chem., 2012, 84, 5140 .

15 M.C. Barr, J.A. Rowehl, R.R. Lunt, J. Xu, A. Wang, C.M Boyce, S.G. Im, V. Bulovic and K.K. Gleason, Adv. Mater., 2011, 23, 3500.

16 M. Müller, C. Czihak, H. Schober, Y. Nishiyama and G. Vogl, Macromolecules, 2000, 33, 1834.

17 M. Österberg, J. Vartiainen, J. Lucenius, U. Hippi, J. Seppälä, R. Serimaa and J. Laine, ACS Appl. Mater. Interfaces, 2013, 5, 4640.

18 K.-Y. Lee, T. Tammelin, K. Schulfter, H. Kiiskinen, J. Samela and A. Bismarck, ACS Appl. Mater. Interfaces, 2012, 4, 4078.

19 M. Henriksson, L.A. Berglund, P. Isaksson, T. Lindström and T. Nishino, Biomacromolecules, 2008, 9, 1579.

20 E. Kontturi, T. Tammelin, M. Österberg, Chem. Soc. Rev., 2006, $35,1287$.

21 C. Aulin, S. Ahola, P. Josefsson, T. Nishino, Y. Hirose, M.; Österberg and L. Wågberg, Langmuir, 2009, 25, 7675.

22 S. Arola, J.-M. Malho, P. Laaksonen, M. Lille and M. Linder, Soft Matter 2013, 91319.

23 B. Belbekhouche, J. Bras, G. Siqueira, C. Chappey, L. Lebrun, B. Khelifi, S. Marais and A. Dufresne, Carbohydr. polym., $2011, \mathbf{8 3}, 1740$.

24 M. Henriksson and L. Berglund, J. Appl. Pol. Sci. 2007, 106, 2817.

25 T.-M. Tenhunen, M.-S. Peresin, P.A. Penttilä, R. Serimaa, J Pere and T. Tammelin. Reactive and Functional Polymers 2014, DOI: 10.1016/j.reactfunctpolym.2014.08.011
26 M.S. Peresin, K. Kammiovirta, H. Setälä and T. Tammelin, $J$. Polym. Environ., 2012, 20, 895.

27 M. Sjögvist, A. Boldizar and M. Rigdahl, J. Cell. Plast., 2010, 46, 497.

28 T. Tammelin, T. Saarinen, M. Österberg and J. Laine, Cellulose, 2006, 13, 519.

29 M. Holmberg, J. Berg, S. Stemme, L. Ödberg, J. Rasmusson and P. Claesson, J. Colloid Interface Sci. 1997, 186, 369.

30 C. Laine, A. Harlin, J. Hartman, S. Hyvärinen, K. Kammiovirta, B. Krogerus, H. Pajari, H. Rautkoski, H. Setälä, J. Sievänen, J. Uotila and M. Vähä-Nissi, Ind. Crop. Prod. 2013, 44, 692.

31 E. Kontturi, P.C. Thüne and J.W. Niemantsverdriet, Langmuir 2003, 19, 5735

32 M. Schaub, G. Wenz, G. Wegner, A. Stein and D. Klemm, $A d v$. Mater., 1993, 5, 919

33 G. Wegner, V. Buchholz, L. Ödberg and S. Stemme, Adv. Mater., 1996, 8, 399.

34 M. Rodahl, F. Höök, A. Krozer, P. Brzezinski and B. Kasemo, Rev. Sci. Instrum., 1995, 66, 3924.

35 F. Höök, M. Rodahl, P. Brzezinski and B. Kasemo, Langmuir, 1998, 14, 729.

36 G. Sauerbrey, Z. Physik. 1959, 155, 206.

37 L. Greenspan, J. Res. Natl. Bureau Stand., 1977, 81, 89.

38 E. Kontturi, M. Suchy, P. Penttilä, B. Jean, K. Pirkkalainen, M. Torkkeli and R. Serimaa, Biomacromolecules, 2011, 12, 770.

39 Kaya, X. Du, Z. Liu, J.W. Lu, J.R. Morris, W.G. Glasser, T. Heinze and A.R. Esker, Biomacromolecule,s 2009, 10, 2451.

40 J. Peltonen, M. Järn, S. Areva, M. Linden and J.B. Rosenholm, Langmuir, 2004, 20, 9428.

41 E. Kontturi, L. Johansson, K.S. Kontturi, P. Ahonen, P.C. Thüne and J. Laine, Langmuir, 2007, 23, 9674.

42 A.R. Urquhardt and A.M. Williams, J. Text. Inst. Trans., 1924, 75, 559.

43 C. W. Vertucci and A. C. Leopold, Plant Physiol. 1987, 85, 232.

44 H. Gocho, A. Tanioka and T. Nakajima, J. Colloid Interface Sci., 1998, 200, 155.

45 H. Gocho, H. Shimizu, A. Tanioka, T.-J. Chou and T. Nakajima, Carbohydrate Polymers, 2000, 41, 87.

46 H. Gocho, H. Shimizu, A. Tanioka, T.-J. Chou and T. Nakajima, Carbohydrate Polymers, 2000, 41, 83.

47 S. Okubayashi, U. J. Griesser and T. Bechtold, Carbohydrate Polymers, 2004, 58, 293.

48 S. Nagarajan and V.K. Pandita, Jour. Agric. Physics., 2005, 5, 11.

49 T. Tammelin, A. Paananen, M. Österberg, In: The Nanoscience and Technology of Renewable Biomaterials, ed. A. L. Lucia and O. J. Rojas, Wiley-Blackwell Publishing Ltd, West Sussex, UK, 2009, pp. 149-172.

50 P. Eronen, M. Österberg, S. Heikkinen, M. Tenkanen and J. Laine, Carbohydr. Polym., 2011, 86, 1281

51 A. Lozhechnikova, D. Dax, J. Vartiainen, S. Willför, C. Xu and M. Österberg, Carbohydr. Polym., 2014, 110, 163.

52 Å. Linder, R. Bergman, A. Bodin and P. Gatenholm, Langmuir, 2003, 19, 5072 
